\title{
COMBINING ABILITY AND HYBRID VIGOR IN INTERSPECIFIC (Gossypium hirsutum L. x Gossypium barbadense L.) LINE X TESTER HYBRIDS OF COTTON
}

\author{
Guven BORZAN $N^{1}$, Ramazan Sadet GUVERCIN ${ }^{2 *}$ \\ 1. East Mediterranean Transitional Zone Agricultural Research Institute, Kahramanmaras, TURKEY \\ 2, Kahramanmaras Sutcu Imam University, Turkoglu Vocational High School, Kahramanmaras, TURKEY \\ *Corresponding author: rguvercin@ksu.edu.tr
}

Received: 30.01.2020

\begin{abstract}
Cotton is most important fiber plant having different fiber properties in the world. This study was carried out to increase yield and ginning outturn of seven interspecific ( $G$ hirsutum $\mathrm{L}$. $\mathrm{x} G$ barbadense $\mathrm{L}$.) pure (F9) lines of cotton. For this, lines were hybridized with Stoneville 468 and Claudia cultivars which belonging to $G$ hirsutum L. genus, in 2018, by Line $x$ Tester mating design. Then, $14 \mathrm{~F}_{1}$ Hybrids were sown with 9 parents according to Randomized Complate Block Design with three replications, in 2019, in Kahramanmaras.

In the results of study, BGKM-3 x Stoneville 468 and BGKM-5 x Claudia $F_{1}$ hybrids had been found very significant for yield and acceptable ginning outturn with good fiber properties. Morever, while BGKM-3 x Stoneville 468 hybrid showed positive for specific combination ability (SCA) in seed cotton yield and ginning outturn, BGKM-3 line showed very important for general combination ability (GCA) in terms of yield and fiber length. Also, Stoneville 468 tester showed important GCA both fiber fineness and ginning outturn.

Inheritance for seed cotton yield have been found higher than other traits and determined both environmental and dominant genes are effective on the seed cotton yield. Besides, contribution of lines on total variance were found higher than both testers and lines $x$ testers interaction, excluding fiber fineness.
\end{abstract}

Keywords: Cotton, fiber properties, interspecific hybrids, Line x Tester, seed cotton yield

\section{INTRODUCTION}

Cotton is a fiber plant having genetic diverse, which produced in the 33.7 million ha area in the world, while in 477800 ha area of Turkey (Anonymous, 2019). 90\% of world cotton production is made with $G$ hirsutum $\mathrm{L}$. (Upland), whilst 3\% with extra long fiber (ELS) $G$ barbadense L. species cotton. $G$ barbadense L genus is known for having fibers which strength, fineness and uniform with longer than $34 \mathrm{~mm}$, in contrast to low seed cotton yield and ginning outturn.

Cotton is very important for Turkish economy and social life. While cotton is production with upland $(G$ hirsutum L.) cultivars in Turkey, ELS cotton is importing from abroad. Because ELS cultivars hasn't adapt to Turkey's conditions. However, because of demand of textile sector continues about quality fiber, breeders have been led to interspecies crosses as a solution. However, although the hybridization between $G$ hirsutum L. and $G$ barbadense L. species began over years ago, there was no success. Because, the ability to combine yield with fiber quality in $\mathrm{F}_{1}$ crosses depends on the genetic potantials and combining ability of the parents (Zhang et al., 2016). The interspecific hybridization studies in Turkey last twenty years have been intensified. Because, interspecific hybrids showed positive heterosis in fiber length, fiber strength (Unay et al., 2018), seed cotton yield (Adsare et al., 2017) and fiber yield (Zhang et al., 2016). However, negative heterobeltiosis was determined for boll number, boll weight (Malathi et al., 2019) and fiber fineness (Gungor and Efe, 2017).

Line $\mathrm{x}$ Tester analysis, help to determine of genetic potential of varieties, is used extensively in Turkey. This analysis method explains the total contribution (GCA) of one parent to crossbreed, while at same time explains the importance of the relationship (SCA) with each of the other parents in crossbreed (Kempthorne, 1957). In addition, parents with high general combination ability are important for pure line development, whereas crosses with high specific combination ability are important in terms of $F_{1}$ hybrid breeding. Because, $\mathrm{F}_{1}$ hybrids can be more superior from mean of parents (heterosis) and superior parent (heterobeltiosis) because of the influence of dominant genes. Previous studies have reported that $F_{1}$ hybrids showed positive heterosis (Naquibullah et al., 2000) and heterobeltiosis in terms of seed cotton yield, ginning outturn, boll weight (Malathi et al., 2019), earliness and some fiber properties. BGKM-3 x Stoneville 468 hybrid 
showed positive heterosis in cotton yield, fiber yield, earliness, fiber length, strength and fineness, but negative heterobeltiosis except for ginning outturn. This situation has been found important for combining high seed cotton yield with good fiber properties in $\mathrm{F}_{1}$ hybrids.

This study was aimed to increase seed cotton yield and ginning outturn of seven pure cotton genotypes $\left(\mathrm{F}_{9}\right)$ which developed by interspecific crossing between $G$ hirsutum L. and $G$ barbadense L. and having thin, long and strength fibers in new hybridization program.

\section{MATERIALS AND METHODS}

This study was conducted in 2018 and 2019 years in Kahramanmaras to improvement seed cotton yield and ginning outturn in seven interspecific ( $G$ hirsutum L. x $G$ barbadense L.) pure lines $\left(\mathrm{F}_{9}\right)$. While interspecific lines (BGKM-1 and BGKM-2 were developed from Sayar $314 \mathrm{x}$ Giza 45, BGKM-3 and BGKM-4 were developed from Maras 92 x Giza 45, BGKM-5, BGKM-6 and BGKM-7 were developed from Ersan $92 \times$ Giza 45) were used as female, Stoneville 468 and Claudia were used as tester parents, and hybridizations were made according to Line $\mathrm{x}$ Tester mating design. Stoneville 468 is preferred by farmers for its high seed cotton yield, while Claudia is preferred for fiber quality. Then, 23 genotypes ( 7 parents and $14 \mathrm{~F}_{1}$ hybrids) were planted at East Mediterranean Transitional Zone Agricultural Research Institute's experimental area according to Randomized Complete Block Design with three replicates in May 22 ${ }^{\text {nd }}, 2019$. The genotypes were sown four rows and $6 \mathrm{~m}$ long in every plot. The inter-row and intra-row of the plants were $0.70 \mathrm{~m}$ and $0.15 \mathrm{~m}$ respectively. $180 \mathrm{~kg} \mathrm{ha}^{-1}$ nitrogen $(\mathrm{N})$ and $60 \mathrm{~kg} \mathrm{ha}^{-}$ 1 phosphorus $\left(\mathrm{P}_{2} \mathrm{O}_{5}\right)$ were applied to experiment area. While first fertilization was applied dose as 60-60-0 NPK $\mathrm{kg} \mathrm{ha}^{-1}$ in the form of 20.20 .0 composed fertilizer before sown, remaining nitrogen $\left(120 \mathrm{~kg} \mathrm{ha}^{-1}\right)$ was applied before first irrigation as ammonium nitrate $(\% 33 \mathrm{~N})$. The all cultural practices were applied according to cotton growing practices of Kahramanmaras. The experimental area was irrigated with 8 times. Harvests were done twice with by hand at October $16^{\text {th }}, 2019$ and November $6^{\text {th }}, 2019$. Fiber characteristics were analyzed with High Volume Instruments (HVI), also. Data of investigated properties were analyzed according to Line $\mathrm{x}$ Tester mating design. Then, means of investigated properties were compared via LSD $_{0.05}$. Furthermore, after the calculated GCA and SCA, heterosis (Chang and Smith, 1967) and heterobeltiosis (Fonseca and Patterson, 1968) were determined by formulas below.

$$
\begin{array}{ll}
\text { Heterosis }(\%) ; & \mathrm{Ht}=\left(\left(\mathrm{F}_{1}-\mathrm{MP}\right) / \mathrm{MP}\right) * 100, \mathrm{MP}=(\text { Line parent+Tester parent }) / 2 \\
\text { Heterobeltiosis }(\%) ; & \left.\mathrm{Htb}=\left(\mathrm{F}_{1} \text {-Better Parent }\right) / \text { Better Parent }\right) * 100
\end{array}
$$

\section{RESULTS AND DISCUSSION}

\section{Line $x$ Tester Analysis and ratio of GCA/SCA}

Results of variance analysis showed that genetic differences among genotypes are important for all properties except fiber strength and uniformity (Table 1). Parents (lines and testers) and hybrids showed important variation for seed cotton yield, ginning outturn, earliness, fiber fineness and fiber length. The significant parents and hybrids indicated that these characters were cotrolled by both additive and non-addtive gene effects. In addition, the variations between lines and testers were positively affected to line $\mathrm{x}$ tester interaction. Furthermore, the negative $\delta_{\mathrm{GCA}}^{2} / \delta^{2} \mathrm{SCA}$ ratios on investigated properties have determined as dominant gene/s effects (Table 1). Previous studies reported that different gene effects in the traits examined. Unay et al. (2019) reported the dominant gene effects on seed cotton yield, ginning outturn fiber length and strength, in contrast, Prakash et al. (2018) and Roy et al. (2018) reported that the additive gene effects are more important.

\section{Performances of Lines}

The averages of lines in seed cotton yield is $3743.0 \mathrm{~kg}$ $\mathrm{ha}^{-1}$, in ginning outturn $36.72 \%$, in fiber yield $1373.8 \mathrm{~kg} \mathrm{ha}^{-}$ 1 , in earliness $70.88 \%$, in fiber length $33.64 \mathrm{~mm}$, in fiber fineness 4.24 micronaire, in fiber strength $36.25 \mathrm{~g} \mathrm{tex}^{-1}$ and in fiber uniformity $86.71 \%$ has been found (Table 2). Differences among lines more effected to variations of fiber yield $(97.67 \%)$, seed cotton yield $(96.28 \%)$, earliness (93.30\%), ginning outturn $(89.25 \%)$, fiber length $(82.42 \%)$, fiber uniformity $(53.74 \%)$, fiber strength $(50.72 \%)$ than testers and lines $x$ testers interaction (Table 4). BGKM-3 line has the highest ( $\left.3951.0 \mathrm{~kg} \mathrm{ha}^{-1}\right)$, but BGKM-1 line has the lowest seed cotton yield ( $\left.3564.3 \mathrm{~kg} \mathrm{ha}^{-1}\right)$ in the study. Moreover, BGKM-3 exceeds the effect of low ginning outturn with its high yield potential and came first in fiber yield. The earliness ratios of lines changed from $69.16 \%$ to $71.97 \%$, and BGKM-6 was determined as earliest genotype. On the other hand, while fiber fineness of lines were varied from 4.16 (BGKM-5) to 4.31 micronaire (BGKM-1), the fiber length $32.87 \mathrm{~mm}$ (BGKM-6) to 34.26 mm (BGKM-3), the fiber strength $37.77 \mathrm{~g} \mathrm{tex}^{-1}$ (BGKM-4) to $35.64 \mathrm{~g} \mathrm{tex}^{-1}$ (BGKM-3) and the fiber uniformity $86.22 \%$ (BGKM-5) to $87.60 \%$ (BGKM-4) varied. 
Table 1. Variance analysis of Line $\mathrm{x}$ Testers for combining effects of different cotton characters

\begin{tabular}{|c|c|c|c|c|c|c|c|c|c|c|c|c|c|c|c|}
\hline Sources & $\begin{array}{l}\text { Degree of } \\
\text { freedom }\end{array}$ & $\begin{array}{c}\text { Seed cotto } \\
\text { yield } \\
\left(\mathrm{kg} \mathrm{ha}^{-1}\right)\end{array}$ & & $\begin{array}{r}\text { Ginnir } \\
\text { outtur } \\
(\%)\end{array}$ & & $\begin{array}{r}\text { Fiber yiel } \\
\left(\mathbf{k g ~ h a}^{-1}\right)\end{array}$ & & $\begin{array}{c}\text { Earlines } \\
(\%)\end{array}$ & & $\begin{array}{c}\text { Fiber } \\
\text { fineness } \\
\text { (micronai }\end{array}$ & & $\begin{array}{r}\text { Fibe } \\
\text { lengt } \\
(\mathbf{m m}\end{array}$ & & $\begin{array}{r}\text { Fiber } \\
\text { strengt) } \\
\left(\mathrm{g} \mathrm{tex}^{-1}\right.\end{array}$ & $\begin{array}{c}\text { Uniformity } \\
\text { ratio } \\
(\%)\end{array}$ \\
\hline Replications & 2 & 117.71 & & 0.22 & & 5.6 & & 0.50 & & 0.007 & & 1.42 & & 3.315 & 0.84 \\
\hline Genotypes & 22 & 4530.35 & $* *$ & 13.47 & $* *$ & 1332.86 & $* *$ & 117.24 & ** & 0.0491 & $* *$ & 3.93 & $* *$ & 6.99 & 33.24 \\
\hline Parents (P) & 8 & 6953.44 & $* *$ & 27.04 & $* *$ & 2853.61 & ** & 210.57 & *** & 0.051 & $* *$ & 4.52 & * & 8.61 & 28.51 \\
\hline Hybrids $(\mathrm{H})$ & 13 & 3277.85 & $* *$ & 4.68 & $* *$ & 441.086 & $*$ & 22.51 & * & 0.043 & * & 3.79 & * & 2.44 & 4.02 \\
\hline $\mathrm{P}$ versus $\mathrm{H}$ & 1 & 1428.06 & & 19.26 & $* *$ & 760.02 & * & 602.06 & *** & 0.11 & * & 1.05 & & 53.4 & 0.71 \\
\hline Lines & 6 & 6838.05 & $* *$ & 9.05 & $* *$ & 933.41 & ** & 45.51 & *** & 0.035 & * & 6.76 & ** & 2.68 & 2.16 \\
\hline Testers & 1 & 986.70 & $*$ & 4.11 & $*$ & 12.86 & & 12.29 & * & 0.32 & $* *$ & 5.54 & & 11.21 & 1.01 \\
\hline $\begin{array}{l}\text { Lines } x \\
\text { Testers }\end{array}$ & 6 & 99.51 & & 0.405 & & 20.13 & & 1.22 & & 0.007 & & 0.52 & & 0.73 & 0.86 \\
\hline Error & 44 & 868.30 & & 1.82 & & 174.87 & & 10.82 & & 0.017 & & 1.55 & & 3.98 & 84.66 \\
\hline$\delta^{2} \mathrm{GCA}$ & & 1470.72 & & 2.04 & & 196.25 & & 0.11 & & 0.02 & & 1.6 & & 0.93 & 0.11 \\
\hline$\delta^{2} \mathrm{SCA}$ & & -256.26 & & -0.47 & & -51.58 & & -0.59 & & -0.0004 & & 0.34 & & -1.09 & -0.59 \\
\hline$\delta^{2}{ }_{\mathrm{GCA}} / \delta^{2} \mathrm{SCA}$ & & -5.74 & & -4.33 & & -3.80 & & -0.18 & & -5.21 & & $4.66^{-}$ & & -0.86 & -0.18 \\
\hline
\end{tabular}

Table 2. Values Line $x$ Tester crosses and parent investigated properties.

\begin{tabular}{|c|c|c|c|c|c|c|c|c|c|c|c|c|c|c|c|}
\hline \multirow{2}{*}{$\begin{array}{l}\text { Crosses } \\
\text { BGKM-1 x Stoneville } 468\end{array}$} & \multicolumn{2}{|c|}{$\begin{array}{c}\text { Seed cotton } \\
\text { yield } \\
\left(\mathrm{kg} \mathrm{ha}^{-1}\right)\end{array}$} & \multicolumn{2}{|c|}{$\begin{array}{l}\text { Ginning } \\
\text { outturn } \\
(\%)\end{array}$} & \multicolumn{2}{|c|}{$\begin{array}{c}\text { Fiber yield } \\
\left(\mathrm{kg} \mathrm{ha}^{-1}\right)\end{array}$} & \multicolumn{2}{|c|}{$\begin{array}{c}\text { Earliness } \\
(\%)\end{array}$} & \multicolumn{2}{|c|}{$\begin{array}{c}\text { Fiber } \\
\text { fineness } \\
\text { (mic.) } \\
\end{array}$} & \multicolumn{2}{|c|}{$\begin{array}{c}\text { Fiber } \\
\text { length } \\
(\mathrm{mm})\end{array}$} & \multicolumn{2}{|c|}{$\begin{array}{c}\text { Fiber } \\
\text { strength } \\
\left(\mathrm{g} \mathrm{tex}^{-1}\right) \\
\end{array}$} & \multirow{2}{*}{$\begin{array}{c}\begin{array}{c}\text { Uniformity } \\
\text { ratio } \\
(\%)\end{array} \\
86.03\end{array}$} \\
\hline & 3702.4 & $\mathrm{e}$ & 38.32 & $\mathrm{c}$ & 1420.0 & & 77.93 & d & 4.36 & abcd & 32.40 & bcd & 32.30 & & \\
\hline BGKM-1 x Claudia & 3666.7 & $\mathrm{e}$ & 39.26 & $\mathrm{c}$ & 1438.6 & & 77.70 & d & 4.23 & d & 33.11 & $a b c$ & 33.50 & & 86.27 \\
\hline BGKM-2 x Stoneville 468 & 4392.9 & $\mathrm{abc}$ & 37.84 & $\mathrm{c}$ & 1659.7 & & 84.93 & bc & 4.35 & bcd & 33.18 & $\mathrm{abc}$ & 32.60 & & 85.40 \\
\hline BGKM-2 x Claudia & 4333.3 & $\mathrm{a}-\mathrm{d}$ & 38.13 & $\mathrm{c}$ & 1652.9 & & 82.71 & $\mathrm{~cd}$ & 4.20 & d & 33.41 & $a b c$ & 33.63 & & 85.93 \\
\hline BGKM-3 x Stoneville 468 & 4750.0 & a & 38.45 & $\mathrm{c}$ & 1826.9 & & 85.58 & $\mathrm{abc}$ & 4.59 & a & 33.58 & $\mathrm{abc}$ & 33.57 & & 86.07 \\
\hline BGKM-3 x Claudia & 4500.0 & $\mathrm{ab}$ & 38.53 & $\mathrm{c}$ & 1735.2 & & 84.20 & bc & 4.29 & bcd & 34.84 & a & 34.10 & & 86.40 \\
\hline BGKM-4 x Stoneville 468 & 3773.8 & de & 39.00 & $\mathrm{c}$ & 1469.2 & & 78.06 & d & 4.41 & abcd & 33.56 & $\mathrm{abc}$ & 32.07 & & 85.97 \\
\hline BGKM-4 x Claudia & 3726.2 & e & 39.30 & $\mathrm{c}$ & 1463.2 & & 77.88 & d & 4.26 & $\mathrm{~cd}$ & 34.32 & ab & 34.40 & & 86.10 \\
\hline BGKM-5 x Stoneville 468 & 4023.8 & b-e & 41.69 & ab & 1679.7 & & 81.44 & $\mathrm{~cd}$ & 4.58 & a & 32.18 & bcd & 34.23 & & 86.33 \\
\hline BGKM-5 x Claudia & 4000.0 & b-e & 41.90 & a & 1679.1 & & 81.23 & $\mathrm{~cd}$ & 4.35 & bcd & 32.31 & bcd & 35.53 & & 86.47 \\
\hline BGKM-6 x Stoneville 468 & 3952.4 & b-e & 38.85 & $\mathrm{c}$ & 1533.1 & & 80.88 & $\mathrm{~cd}$ & 4.48 & $\mathrm{abc}$ & 31.90 & $\mathrm{~cd}$ & 33.50 & & 85.87 \\
\hline BGKM-6 x Claudia & 3845.2 & cde & 40.10 & $\mathrm{abc}$ & 1542.1 & & 78.71 & d & 4.37 & bcd & 32.17 & bcd & 34.17 & & 85.80 \\
\hline BGKM-7 x Stoneville 468 & 4226.2 & $\mathrm{a}-\mathrm{e}$ & 38.26 & $\mathrm{c}$ & 1617.6 & & 82.88 & $\mathrm{~cd}$ & 4.49 & ab & 30.41 & d & 33.83 & & 85.63 \\
\hline \multicolumn{16}{|l|}{ Lines } \\
\hline BGKM-1 & 3564.3 & $\mathrm{~b}$ & 38.50 & $\mathrm{~b}$ & 1372.8 & bc & 71.27 & $\mathrm{e}$ & 4.31 & $\mathrm{~b}$ & 33.79 & $\mathrm{a}$ & 35.83 & $\mathrm{a}$ & 86.27 \\
\hline BGKM-2 & 3906.0 & $\mathrm{~b}$ & 36.10 & $\mathrm{c}$ & 1410.7 & & 70.93 & e & 4.27 & $\mathrm{~b}$ & 33.91 & a & 36.40 & a & 86.62 \\
\hline BGKM-3 & 3951.0 & $\mathrm{~b}$ & 36.76 & bc & 1450.9 & b & 71.74 & $\mathrm{e}$ & 4.21 & $\mathrm{~b}$ & 34.26 & a & 37.77 & a & 86.87 \\
\hline BGKM-4 & 3637.6 & $\mathrm{~b}$ & 37.36 & bc & 1359.8 & bc & 70.88 & e & 4.26 & $\mathrm{~b}$ & 33.66 & $\mathrm{ab}$ & 35.64 & a & 87.60 \\
\hline BGKM-5 & 3717.9 & $\mathrm{~b}$ & 35.27 & $\mathrm{c}$ & 1310.7 & $\mathrm{c}$ & 69.16 & $\mathrm{e}$ & 4.16 & $\mathrm{~b}$ & 33.74 & a & 36.47 & a & 86.22 \\
\hline BGKM-6 & 3650.0 & $\mathrm{~b}$ & 35.78 & $\mathrm{c}$ & 1305.0 & $\mathrm{c}$ & 71.97 & e & 4.24 & $\mathrm{~b}$ & 32.87 & $a b$ & 35.83 & a & 86.82 \\
\hline BGKM-7 & 3774.5 & $\mathrm{~b}$ & 37.27 & $\mathrm{bc}$ & 1406.5 & $\mathrm{bc}$ & 70.24 & $\mathrm{e}$ & 4.25 & $\mathrm{~b}$ & 33.23 & $\mathrm{ab}$ & 35.80 & ab & 86.59 \\
\hline \multicolumn{16}{|l|}{ Testers } \\
\hline Stoneville 468 & 4904.8 & $\mathrm{a}$ & 42.53 & $\mathrm{a}$ & 2085.5 & $\mathrm{a}$ & 90.72 & $\mathrm{a}$ & 4.61 & $\mathrm{a}$ & 30.45 & $\mathrm{c}$ & 32.11 & $\mathrm{c}$ & 83.73 \\
\hline Claudia & 4675.0 & a & 43.75 & a & 2044.1 & a & 88.80 & ab & 4.34 & $\mathrm{~b}$ & 31.76 & $\mathrm{bc}$ & 33.43 & $\mathrm{bc}$ & 85.63 \\
\hline Means of crosses & 4068.9 & 39.23 & 1595.4 & 81.13 & 4.38 & 32.82 & 33.67 & 86.06 & & & & & & & \\
\hline Means of lines & 3743.0 & 36.72 & 1373.8 & 70.88 & 4.24 & 33.64 & 36.25 & 86.71 & & & & & & & \\
\hline Means of testers & 4789.9 & 43.14 & 2064.8 & 89.76 & 4.48 & 31.11 & 32.77 & 84.68 & & & & & & & \\
\hline Means of parents & 4266.5 & 39.93 & 1719.3 & 80.32 & 4.36 & 32.37 & 34.51 & 85.70 & & & & & & & \\
\hline Means of genotypes & 4167.7 & 39.58 & 1657.3 & 80.59 & 4.37 & 32.6 & 34.09 & 85.88 & & & & & & & \\
\hline $\mathrm{LSD}_{\text {Hybrids }}$ & $60.08^{* *}$ & $2.28^{*}$ & $\begin{array}{r}26.91 \\
\text { ns }\end{array}$ & $\begin{array}{l}5.35 \\
* *\end{array}$ & $2.17^{*}$ & $0.22^{*}$ & $\begin{array}{r}3.91 \\
\text { ns }\end{array}$ & $\begin{array}{l}2.42 \\
\mathrm{~ns}\end{array}$ & & & & & & & \\
\hline $\mathrm{LSD}_{\text {Parents }}$ & $33.18^{* *}$ & $2.31^{* *}$ & $13.12^{* *}$ & 4.96 & $0.22^{*}$ & $1.93^{* *}$ & $2.34^{* *}$ & $\begin{array}{l}2.32 \\
\text { ns }\end{array}$ & & & & & & & \\
\hline $\mathrm{CV}(\%)$ & 12.81 & 3.51 & 10.96 & 3.81 & 2.91 & 3.38 & 3.81 & 1.56 & & & & & & & \\
\hline
\end{tabular}

LSD: Least significant degrees, CV: Coefficient of variation, *; $P<0.05$, **: $P<0.01$, ns: Non-significant

\section{Performances of Testers}

The averages of testers have found as $4789.9 \mathrm{~kg} \mathrm{ha}^{-1}$ in seed cotton yield, $43.14 \%$ in ginning outturn, $2064.8 \mathrm{~kg} \mathrm{ha}$ ${ }^{1}$ in fiber yield, $89.76 \%$ in earliness, $31.11 \mathrm{~mm}$ fiber length, 4.48 micronaire in fiber fineness, $32.77 \mathrm{~g} \mathrm{tex}^{-1}$ in fiber strength and $84.68 \%$ in fiber uniformity. The variation among testers contributed $55.97 \%$ to fiber fineness, $35.42 \%$ to fiber strength, $25.00 \%$ to fiber uniformity, $11.26 \%$ to fiber length, $6.76 \%$ to ginning outturn, $4.20 \%$ to earliness, $2.32 \%$ to seed cotton yield and $0.22 \%$ to fiber yield (Table 4). In the study, while Stoneville 468 was determined as more productive with earliness and coarse fiber, Claudia 
was found other important tester in terms of ginning outturn and fiber properties (Table 2).

\section{Performances of Line $x$ Tester Hybrids}

Averages of hybrids were determined as $4068.9 \mathrm{~kg} \mathrm{ha}^{-1}$ in seed cotton yield, $39.23 \%$ ginning outturn, $1595.4 \mathrm{~kg} \mathrm{ha}^{-}$ ${ }^{1}$ in fiber yield, $81.13 \%$ in earliness, 4.38 micronaire in fiber fineness, $32.82 \mathrm{~mm}$ in fiber length, $33.67 \mathrm{~g} \mathrm{tex}^{-1}$ in fiber strength, and $86.06 \%$ in fiber uniformity. Furthermore, Line $\mathrm{x}$ Tester interaction contributed to the variations $1.40 \%$ in seed cotton yield, $4.00 \%$ in ginning outturn, $2.10 \%$ in fiber yield, $2.50 \%$ in earliness, $7.07 \%$ in fiber fineness, $6.32 \%$ in fiber length, $13.86 \%$ in fiber strength and $21.27 \%$ in fiber uniformity (Table 4). Among the hybrid, BGKM-3 x Stoneville $468 \mathrm{~F}_{1}$ has obtained high cotton yield (4750.0 $\left.\mathrm{kg} \mathrm{ha}^{-1}\right)$, fiber yield $\left(1826.9 \mathrm{~kg} \mathrm{ha}^{-1}\right)$ and earliness $(85.58 \%)$. Moreover, BGKM-5 x Claudia $\mathrm{F}_{1}$ was getting highest ginning outturn (41.90\%), while BGKM-3 x Claudia $F_{1}$ for fiber length $(34.84 \mathrm{~mm})$ and BGKM-5 x Claudia $F_{1}$ for fiber strength $\left(35.53 \mathrm{~g} \mathrm{tex}^{-1}\right)$ and uniformity $(86.47 \%)$ had been found very important. Basal et al. (2017) were reported that interspecies (G barbadense
L. x G hirsutum L.) crosses showed negative GCA in fiber quality, while positive GCA in seed cotton yield.

\section{GCA of Parents}

According to Table 3 , lines showed positive GCA on some properites. While BGKM-2 shows significant GCA in seed cotton yield $(29.42 *)$ and BGKM-5 in ginning outturn $\left(2.57^{* *}\right)$, BGKM-3 is showed very significant and positive GCA in seed cotton yield (55.61**), in fiber yield $(18.57 * *)$, in earliness $(3.76 * *)$ and in fiber length $(1.39 * *)$. While BGKM-3 line would be most important parent due to positive contribution to six traits, showing insignificant and negative GCA (-0.74) in term of ginning outturn had been attracted attention. On the other hand, testers showed negligible GCA in other traits, except fiber fineness. Stoneville 468 showed positive GCA in seed cotton yield (4.85), in fiber yield (0.55), in earliness (0.54) and in fiber fineness $\left(0.09^{* *}\right)$, while Claudia had been showed positive GCA in term of ginning outturn, fiber length, fiber strength, and fiber uniformity properties.

Table 3. The combining ability Line $x$ Tester crosses (SCA) and parents (GCA) in studied traits

\begin{tabular}{|c|c|c|c|c|c|c|c|c|}
\hline Crosses & $\begin{array}{c}\text { Seed cotton } \\
\text { yield } \\
\left(\mathrm{kg} \mathrm{ha}^{-1}\right)\end{array}$ & $\begin{array}{c}\text { Ginning } \\
\text { outturn } \\
(\%)\end{array}$ & $\begin{array}{c}\text { Fiber } \\
\text { yield } \\
\left(\mathrm{kg} \mathrm{ha}^{-1}\right)\end{array}$ & $\begin{array}{l}\text { Earliness } \\
(\%)\end{array}$ & $\begin{array}{c}\text { Fiber } \\
\text { fineness } \\
\text { (mic.) }\end{array}$ & $\begin{array}{c}\text { Fiber } \\
\text { length } \\
(\mathrm{mm})\end{array}$ & $\begin{array}{c}\text { Fiber } \\
\text { strength } \\
\left(\mathrm{g} \mathrm{tex}^{-1}\right) \\
\end{array}$ & $\begin{array}{c}\text { Uniformity } \\
\text { ratio } \\
(\%)\end{array}$ \\
\hline $\begin{array}{l}\text { BGKM-1 x Stoneville } \\
468\end{array}$ & -3.06 & -0.16 & -1.48 & -0.42 & 0.01 & -0.02 & -0.08 & 0.04 \\
\hline BGKM-1 x Claudia & 3.06 & 0.16 & 1.48 & 0.42 & -0.01 & 0.02 & 0.08 & -0.04 \\
\hline $\begin{array}{l}\text { BGKM-2 x Stoneville } \\
468\end{array}$ & -1.87 & 0.17 & -0.22 & 0.57 & 0.25 & -0.01 & 0.00 & -0.11 \\
\hline BGKM-2 x Claudia & 1.87 & -0.17 & 0.22 & -0.57 & -0.25 & 0.01 & 0.00 & 0.11 \\
\hline $\begin{array}{l}\text { BGKM-3 x Stoneville } \\
468\end{array}$ & 7.65 & 0.27 & 4.03 & 0.15 & -0.27 & 0.06 & 0.25 & -0.01 \\
\hline BGKM-3 x Claudia & -7.65 & -0.27 & -4.03 & -0.15 & 0.27 & -0.06 & -0.25 & 0.01 \\
\hline $\begin{array}{l}\text { BGKM-4 x Stoneville } \\
468\end{array}$ & -2.47 & 0.16 & -0.26 & -0.45 & -0.02 & -0.01 & -0.65 & 0.09 \\
\hline BGKM-4 x Claudia & 2.47 & -0.16 & 0.26 & 0.45 & 0.02 & 0.01 & 0.65 & -0.09 \\
\hline $\begin{array}{l}\text { BGKM-5 x Stoneville } \\
468\end{array}$ & -3.66 & 0.21 & -0.52 & -0.43 & 0.30 & 0.03 & -0.13 & 0.09 \\
\hline BGKM-5 x Claudia & 3.66 & -0.21 & 0.52 & 0.43 & -0.30 & -0.03 & 0.13 & -0.09 \\
\hline $\begin{array}{l}\text { BGKM-6 x Stoneville } \\
468\end{array}$ & 0.51 & -0.31 & -1.00 & 0.54 & 0.23 & -0.03 & 0.18 & 0.19 \\
\hline BGKM-6 x Claudia & -0.51 & 0.31 & 1.00 & -0.54 & -0.23 & 0.03 & -0.18 & -0.19 \\
\hline $\begin{array}{l}\text { BGKM-7 x Stoneville } \\
468\end{array}$ & 2.89 & -0.34 & -0.55 & 0.05 & -0.49 & -0.01 & 0.43 & -0.28 \\
\hline $\begin{array}{l}\text { BGKM-7 x Claudia } \\
\text { Lines }\end{array}$ & -2.89 & 0.34 & 0.55 & -0.05 & 0.49 & 0.01 & -0.43 & 0.28 \\
\hline BGKM-1 & -38.44 & -0.44 & -16.61 & -3.32 & -0.08 & -0.07 & -0.77 & 0.10 \\
\hline BGKM-2 & $29.42^{*}$ & -1.25 & 6.1 & 2.69 & -0.10 & 0.48 & -0.56 & -0.39 \\
\hline BGKM-3 & $55.61^{* *}$ & -0.74 & $18.57^{* *}$ & $3.76^{* *}$ & 0.06 & $1.39^{* *}$ & 0.16 & 0.18 \\
\hline BGKM-4 & -31.89 & -0.08 & -12.92 & -3.16 & -0.04 & $1.12^{*}$ & -0.44 & -0.02 \\
\hline BGKM-5 & -5.70 & $2.57^{* *}$ & 8.40 & 0.21 & 0.09 & -0.58 & 1.21 & 0.35 \\
\hline BGKM-6 & -17.01 & 0.25 & -5.77 & -1.33 & 0.05 & -0.79 & 0.16 & -0.22 \\
\hline BGKM-7 & 7.99 & -0.31 & 2.23 & 1.16 & 0.03 & -1.55 & 0.24 & 0.01 \\
\hline \multicolumn{9}{|l|}{ Testers } \\
\hline Stoneville 468 & 4.85 & -0.31 & 0.55 & 0.54 & $0.09^{* *}$ & -0.36 & -0.52 & -0.15 \\
\hline Claudia & -4.85 & 0.31 & -0.55 & -0.54 & -0.09 & 0.36 & 0.52 & 0.15 \\
\hline
\end{tabular}


Table 4. Contribution of genotypes to the total variance of the traits studied (\%)

\begin{tabular}{lccc}
\hline Properties & Lines & Testers & Lines x Testers \\
\hline Seed cotton yield & 96.28 & 2.32 & 1.40 \\
Ginning outturn & 89.25 & 6.76 & 4.00 \\
Fiber yield & 97.67 & 0.22 & 2.10 \\
Earliness & 93.30 & 4.20 & 2.50 \\
Fiber fineness & 36.96 & 55.97 & 7.07 \\
Fiber length & 82.42 & 11.26 & 6.32 \\
Fiber strength & 50.72 & 35.42 & 13.86 \\
Uniformity ratio & 53.74 & 25.00 & 21.27 \\
\hline
\end{tabular}

\section{SCA of Hybrids}

In the study, BGKM-1 x Stoneville $468 \mathrm{~F}_{1}$ and BGKM$1 \times$ Claudia $F_{1}$ showed significant and positive SCA in two and six traits, respectively (Table 3). BGKM-2 x Stoneville $468 \mathrm{~F}_{1}$ had positive $\mathrm{SCA}$ in ginning outturn (0.17), earliness (0.57) and fiber length, while BGKM-2 x Claudia $F_{1}$ showed positive SCA in seed cotton yield (1.87), in fiber yield (0.22), in fiber fineness (0.01) and in fiber uniformity (0.11). The BGKM-3 x Stoneville $468 \mathrm{~F}_{1}$ having most seed cotton yield had been showed positive SCA in seed cotton yield (7.65), in ginning outturn (0.27), in fiber yield (4.03), in earliness $(0.15)$, in fiber fineness $(0.06)$ and in fiber length. Moreover, BGKM-3 x Stoneville $468 \mathrm{~F}_{1}$ had combined seed cotton yield, fiber yield and earliness with fiber properties. Besides, dominant gen effects on traits indicated the importance of the BGKM-3 x Stoneville 468 $F_{1}$ for develop both hybrid and conventional variety, while the BGKM-3 x Claudia $F_{1}$ had been found important for development of conventional cultivars. In the study, BGKM-4 x Claudia $F_{1}$ was showed positive SCA in seed cotton yield (2.47), in fiber yield (0.26), in earliness (0.45), in fiber fineness (0.01), in fiber length (0.02) and in fiber strength (0.65), while BGKM-4 x Stoneville $468 \mathrm{~F}_{1}$ was showed insignificant but positive SCA in ginning outturn (0.16) and in fiber uniformity. BGKM-5 x Stoneville 468 and BGKM-5 x Claudia $\mathrm{F}_{1}$ having the highest ginning outturn were showed positive SCA at four traits, and are found important for develop conventional cultivars. BGKM-6 $\mathrm{x}$ Stoneville $468 \mathrm{~F}_{1}$ was found to be more important than BGKM- 6 x Claudia $F_{1}$ with having positive SCA in five traits. BGKM-7 $x$ Claudia $F_{1}$ had been determined more important than BGKM-7 x ST $468 \mathrm{~F}_{1}$. BGKM-7 x Claudia $\mathrm{F}_{1}$ showed insignificant-positive SCA in fiber yield (0.55), in ginning outturn (0.34), in fiber length (0.49), in fiber uniformity (0.19) and fineness (0.01), while BGKM-7 x Stoneville $468 \mathrm{~F}_{1}$ had been showed insignificant-positive SCA in cotton yield (2.89), in fiber strength (0.43) and in earliness (0.05).

\section{Heterotic Effects (Heterosis and Heterobeltiosis)}

In the study, one hybrid in fiber yield, two hybrid in fiber strength, three hybrid in seed cotton yield and ginning outturn, eight hybrid in earliness and fiber fines, ten hybrid in fiber length, and eleven hybrid combinations in fiber uniformity have been showed positive heterosis. In addition, while two hybrids showed positive both heterosis and heterobeltiosis for fiber length, one hybrid showed positive both heterosis and heterobeltiosis in fiber uniformity. BGKM-3 x Stoneville 468 and BGKM-5 x Stoneville $468 \quad F_{1}$ showed positive heterosis in six properties. The four combinations of BGKM-3 and BGKM-5 lines with testers showed positive heterosis in earliness, fiber fineness, fiber length and fiber uniformity, while differentiated in seed cotton yield and ginning outturn. In addition, hybrids of BGKM-3 and BGKM-4 with Claudia showed positive both heterosis and heterobeltiosis in fiber length. Heterosis values of hybrid in seed cotton yield between $-11.66 \%$ (BGKM-4 x Stoneville 468) and 7.29\% (BGKM-3 x Stoneville 468), in ginning outturn between $-5.43 \%$ (BGKM-1 x Stoneville 468) and $7.26 \%$ (BGKM-5 x Stoneville 468), between in fiber yield of $-15.75 \%$ (BGKM-1 x Claudia) and $3.35 \%$ (BGKM-3 x Stoneville 468), in earliness between $-3.44 \%$ (BGKM-1 $\mathrm{x}$ Stoneville 468) $5.38 \%$ (BGKM-3 x Stoneville 468), in fiber fineness between \%-2.35 (BGKM-1 x Stoneville 468) and 4.59\% (BGKM-5 x Stoneville 468), in fiber length between $-4.49 \%$ (BGKM-7 x Stoneville 468) and 5.54\% (BGKM-3 $\mathrm{x}$ Claudia), in fiber strength between $-5.25 \%$ (BGKM-4 $\mathrm{x}$ Stoneville 468) and $1.82 \%$ (BGKM-5 x Claudia), in fiber uniformity between $-0.60 \%$ (BGKM-4 x Claudia) and $1.60 \%$ (BGKM-5 x Stoneville 468) were changed (Table 5). Adsare et al. (2017) found positive heterosis in seed cotton yield, whereas Gungor and Efe (2017) determined positive heterosis in fiber length, fiber strength, fiber fineness and fiber uniformity. Moreover, Coban and Unay (2017) reported positively-significant useful heterosis for fiber strength and length whereas mostly low-negative values for fiber quality parameters were determined by Dhamayanthi (2011). In addition, the heterobeltiosis values of the hybrids have been found positive, in contrast to others, as $1.76 \%$ (BGKM-3 x Claudia) and $1.93 \%$ (BGKM4 x Claudia) in fiber length, as $0.39 \%$ in fiber fineness and as $0.32 \%$ (BGKM-5 x Claudia) in uniformity. In previous studies, Gohil et al. (2017) was detected significant heterobeltiosis in terms of seed cotton yield $(-28.59 \%$ and $31.64 \%)$ and fiber yield $(-37.27 \%$ and $27.04 \%)$, while Gungor and Efe (2017) detected in terms of in fiber length $(1.39 \%)$ and fiber uniformity (-0.62\%). 
Table 5. Heterosis and heterobeltiosis values of line $\mathrm{x}$ tester crosses (\%)

\begin{tabular}{|c|c|c|c|c|c|c|c|c|c|c|c|c|c|c|c|c|}
\hline \multirow[t]{2}{*}{ Crosses } & \multicolumn{2}{|c|}{$\begin{array}{l}\text { Seed cotton yield } \\
\quad\left(\mathrm{kg} \mathrm{ha}^{-1}\right)\end{array}$} & \multicolumn{2}{|c|}{$\begin{array}{l}\text { Ginning outturn } \\
(\%)\end{array}$} & \multicolumn{2}{|c|}{$\begin{array}{l}\text { Fiber yield } \\
\left(\mathrm{kg} \mathrm{ha}^{-1}\right)\end{array}$} & \multicolumn{2}{|c|}{$\begin{array}{l}\text { Earliness } \\
(\%)\end{array}$} & \multicolumn{2}{|c|}{$\begin{array}{c}\text { Fiber } \\
\text { fineness } \\
\text { (micronaire) }\end{array}$} & \multicolumn{2}{|c|}{$\begin{array}{c}\text { Fiber } \\
\text { length } \\
(\mathbf{m m})\end{array}$} & \multicolumn{2}{|c|}{$\begin{array}{c}\text { Fiber } \\
\text { strength } \\
\left(\mathrm{g} \mathrm{tex}^{-1}\right) \\
\end{array}$} & \multicolumn{2}{|c|}{$\begin{array}{c}\text { Uniformity } \\
\text { ratio } \\
(\%)\end{array}$} \\
\hline & Htr & Htb & Htr & Htb & Htr & Htb & Htr & Htb & Htr & Htb & Htr & Htb & Htr & Htb & Htr & Htb \\
\hline BGKM-1 x Stoneville 468 & -12.15 & -24.30 & -5.43 & -9.80 & -17.47 & $-31.64^{*}$ & -3.44 & -13.95 & -2.35 & -5.46 & 0.95 & -4.02 & -4.76 & -9.69 & 1.22 & -0.25 \\
\hline BGKM-1 x Claudia & $-10.99^{* *}$ & $-21.48^{*}$ & -4.44 & -10.22 & $-15.75^{*}$ & $-29.59^{* *}$ & -2.89 & $-12.48 *$ & -2.18 & -2.75 & 1.13 & -1.89 & -3.04 & -6.26 & 0.37 & -0.53 \\
\hline BGKM-2 x Stoneville 468 & -0.21 & -10.23 & -3.7 & $-10.97^{*}$ & -5.15 & $-20.41^{*}$ & 5.30 & -6.19 & $-1.98^{*}$ & -5.58 & 3.22 & -1.81 & $-4.86^{*}$ & $-10.39^{*}$ & 0.27 & -1.39 \\
\hline BGKM-2 x Claudia & 1.04 & -7.18 & -4.50 & $-12.87^{* *}$ & -4.24 & $-19.1^{*}$ & 3.55 & -6.80 & -2.35 & -4.10 & 1.84 & -1.25 & -3.60 & -7.47 & -0.2 & -1.29 \\
\hline BGKM-3 x Stoneville 468 & 7.29 & -3.02 & -2.90 & -9.51 & 3.35 & -12.25 & 5.38 & -5.56 & 4.07 & -0.31 & 3.78 & -1.99 & -3.93 & -11.00 & 0.90 & -0.93 \\
\hline BGKM-3 x Claudia & 4.48 & -3.64 & -4.27 & -11.86 & -0.65 & -15.06 & 4.92 & -5.16 & 0.42 & -1.78 & 5.54 & 1.76 & -4.21 & $-9.70^{* *}$ & 0.20 & -0.58 \\
\hline BGKM-4 x Stoneville 468 & -11.66 & $-23.12^{* *}$ & -2.38 & -8.25 & $-14.70 *$ & $-29.52^{* *}$ & -3.37 & -13.85 & -0.68 & -4.42 & 4.73 & -0.29 & -5.25 & -9.78 & 0.36 & -1.86 \\
\hline BGKM-4 x Claudia & -10.09 & -20.07 & -3.07 & -10.10 & -13.84 & $-28.36^{*}$ & -2.40 & -12.36 & -0.91 & -2.46 & 4.90 & 1.93 & -0.57 & -4.03 & -0.60 & -1.71 \\
\hline BGKM-5 x Stoneville 468 & -6.47 & -17.7 & 7.26 & -1.91 & -0.88 & -19.10 & 1.94 & -10.17 & 4.59 & -0.48 & 0.28 & -4.60 & 0.06 & -5.85 & 1.60 & -0.08 \\
\hline BGKM-5 x Claudia & -4.70 & $-14.46^{*}$ & 6.04 & -4.11 & -0.01 & -17.89 & 2.85 & $-8.48^{*}$ & 2.46 & 0.39 & -1.34 & -4.25 & 1.82 & -2.50 & 0.66 & 0.32 \\
\hline BGKM-6 x Stoneville 468 & -7.54 & -19.16 & -0.74 & -8.61 & -9.52 & $-26.35^{*}$ & -0.48 & $-10.80^{*}$ & 1.09 & -2.96 & 0.75 & -2.87 & -1.41 & $-6.51^{* *}$ & 0.69 & -1.08 \\
\hline BGKM-6 x Claudia & $-7.56^{*}$ & $-17.68^{*}$ & 0.92 & -8.27 & $-7.93^{*}$ & $-24.57^{* *}$ & -2.07 & $-11.32^{*}$ & 1.81 & -0.68 & -0.32 & -1.94 & -1.37 & -4.73 & -0.5 & -1.18 \\
\hline BGKM-7 x Stoneville 468 & -2.60 & -13.83 & -4.06 & $-9.99^{*}$ & -7.28 & $-22.37^{*}$ & 3.02 & -8.57 & 1.41 & -2.52 & -4.49 & $-8.49^{* *}$ & -0.29 & -5.47 & 0.55 & -1.10 \\
\hline BGKM-7 x Claudia & -3.45 & -12.40 & -2.34 & $-9.58^{* *}$ & -6.13 & -20.68 & 2.75 & $-7.99^{* *}$ & 0.96 & -0.10 & -1.15 & -3.64 & -1.84 & -5.04 & 0.47 & -0.15 \\
\hline
\end{tabular}




\section{CONCLUSION}

The lines had been found very weaker than testers and crosses in terms of seed cotton yield and ginning outturn, but superior from than in terms of fiber properties. Testers have been the most productive varieties. Crosses are placed between parents in terms of yield and fiber quality. Lines were more contributed to the inheritance of traits than the testers and lines $\mathrm{x}$ testers interaction, except fiber fineness. While lines gave the highest contribution to fiber yield variation (97.67\%), incontranst, gave the lowest contribution to fiber fineness variation $(36.96 \%)$. BGKM3 , one of the lines, is showed very significant and positive GCA in terms of cotton yield, fiber yield, earliness and fiber length except for ginning outturn, while positive GCA showed in fiber fineness, fiber strength and fiber uniformity. Stoneville 468, one of the testers, was showing as smilarity with Unay et al. (2019) for cotton yield, fiber yield and earliness, Claudia is showed smilarity again in term of ginning outturn, fiber length, fiber fineness, fiber strength and fiber uniformity again with Unay et al. (2019). On the other hand, BGKM-3 x Stoneville $468 \mathrm{~F}_{1}$ hybrid combined seed cotton yield with fiber quality, and was showed positive heterosis in seed cotton yield, fiber yield, earliness, fiber length and fiber uniformity. BGKM-3 x Claudia and BGKM-4 x Claudia $F_{1}$ hybrids have been showed positive heterosis and heterobeltiosis with the effect of dominant gene/s in fiber length. As a result, hybrids have higher seed cotton yield and ginning outturn than lines and BGKM-3 x Stoneville $468 \mathrm{~F}_{1}$ hybrid have been found important not only as $F_{1}$ hybrid but also for conventional cultivar development studies. Because, it produced more seed cotton yield and ginning outturn with fiber quality from BGKM-3 parent.

\section{ACKNOWLEDGEMENT}

The present study was funded by TAGEM (Republic of Turkey Ministry of Agriculture and Forestry General Directorate of Agricultural Research and Policies) with a project number of TAGEM/17/A07/P5/02-01-13. Authors wish to thanks to TAGEM for the financial support.

\section{LITERATURE CITED}

Adsare, A.D., A.N. Salve and N.P. Patil. 2017. Heterosis studies for quantitative traits in interspecific hybrids of cotton $(G$ hirsutum L. x G barbadense L.). Journal of Phytology 9: 1114.

Anonymous, 2019. T.C. Ministry of Trade, General Directorate of Tradesmen, Craftsmen and Cooperatives. Cotton Report. Ankara.

Basal, H., B. Demirok, T. Karahan, E. Ilker and H. Gungor. 2017. Comparison of mean yield components and fiber quality parameters of advanced bulk generations in $F_{2}, F_{3}$ and $F_{4}$ interspecific and intraspecific cotton populations. Turkish Journal of Field Crops 22 (1): 14-23.

Chang M.S. and J.D. Smith. 1967. Diallel Analysis of Inheritance of Quantitative Characters in Grain Sorghum. I. Heterosis and Inbreding Depression. Canadian Journal of Genetics and Cytology 9 (1): 44-51.

Coban, M. and A. Unay. 2017. Gene action and useful heterosis in interspecific cotton crosses ( $G$ hirsutum L. x G barbadense L.). Journal of Agricultural Sciences 23: 438-443.

Dhamayanthi, K.P.M. 2011. Study of interspecific hybrids ( $G$ hirsutum x $G$ barbadense L.) for heterosis and combining ability. World Cott. Res. Conf.-5., Mumbai, India. 51-55.

Fonseca S.M.and F.L. Patterson. 1968. Hybrid Vigor in a Seven Parent Diallel Cross in Common Winter Wheat (T. Aestivum L.). Crop Science 8 (1): 85-88.

Gohil, S.B., M.B. Parmar and D.J. Chaudhari. 2017. Study of heterosis in interspecific hybrids of cotton ( $G$ hirsutum L. x $G$ barbadense L.). Journal of Pharmacognosy and Phytochemistry 6 (4): 804-810.

Gungor, H. and L. Efe. 2017. A heterosis study for fiber quality traits in cotton. Journal of Natural Science 20 (1): 54-66. DOI : 10.18016/ksujns.65079.

Kempthorne, O. 1957. An Introduction to Genetic Statistics. John Wiley and Sons. Inc., New York, U.S.A

Malathi, S., R.S. Patil and H.S. Saritha. 2019. Heterosis studies in interspecific cotton hybrids ( $G$ hirsutum L. x $G$ barbadense L.) under irrigated condition. Electronic Journal of Plant Breeding 10 (2): 852-861.

Naquibullah, H., K. Abro, M.B. Kumbhar, G. Hassan and G. Mahmood. 2000. Study of heterosis in upland cotton - II. Morphology and yield traits. Pak. Cottons. 44:13-23.

Prakash, G., S.L. Korekar. and S. Mankare. 2018. Combining ability analysis in bt cotton ( $G$ hirsutum L.) to harness high yield under contrasting planting densities through heterosis breeding. International Journal of Current Microbiology and Applied Sciences 7: 1765-1774.

Roy, U., M.C. Paloti, R.S. Patil and I.S. Katageri. 2018. Combining ability analysis for yield and yield attributing traits in interspecific ( $G$ hirsutum L. x $G$ barbadense L.) Hybrids of Cotton. Electronic Journal of Plant Breeding 9: 458-464.

Unay, A., D. Altintas and M. Coban. 2018. The determination of leaf anatomy, yield and quality characteristics in $F_{1}$ and $F_{2}$ generations of interspecific cotton hybrids ( $G$ hirsutum L. x $G$ barbadense L.). Turkish Journal of Field Crops 23 (2): 146150.

Unay, A., N. Ozbek and V.M. Cinar. 2019. Line x Tester analysis for yield and fiber quality in cotton ( $G$ hirsutum L.). Turkish Journal of Field Crops 24 (2): 215-220, DOI: $10.17557 /$ tjfc. 509134 .

Zhang, J., M. Wu. J. Yu, X. Li and W. Pei. 2016. Breeding potential of introgression lines developed from interspecific crossing between upland cotton $G$ hirsutum L. and $G$ barbadense L.: heterosis, combining ability and genetic effects. PLoS ONE 11 (1): e0143646. doi:10.1371/journal. pone. 0143646 . 